\title{
Paramètres démographiques et productivité des élevages bovins de la Guinée-Bissau
}

\author{
Mamadou IRA ${ }^{1,2^{*}}$, Guiguigbaza Kossigan DAYO ${ }^{1}$, Mamadou SANGARE ${ }^{1}$, \\ Bacar DJASSI $^{3}$, Joao GOMES ${ }^{3}$, Bernado CASSAMA ${ }^{3}$, Aboubacar TOGUYENI ${ }^{1,2}$, \\ Chia Valentine YAPI-GNAORE ${ }^{1}$ et Georges Anicet OUEDRAOGO ${ }^{2}$ \\ ${ }^{I}$ Centre International de Recherche - Développement sur l'Elevage en zone Subhumide (CIRDES), 01 BP 454 \\ Bobo-Dioulasso 01, Burkina Faso. \\ ${ }^{2}$ Université Nazi Boni de Bobo-Dioulasso (UNB), 01 BP 1091 Bobo-Dioulasso 01, Burkina Faso. \\ ${ }^{3}$ Direction Générale de l'Elevage (DGE), BP 26 Bissau, Guinée-Bissau. \\ * Auteur correspondant ; E-mail : iramad2020@yahoo.fr; Tel: +22676878746/22660616760
}

\section{REMERCIEMENTS}

Cette étude a été réalisée grâce au Projet de gestion et de valorisation des ressources génétiques animales et aquacoles dans l'espace UEMOA (PROGEVAL) financé par la Convention entre le CORAF/WECARD et l'UEMOA. Les travaux de thèse de doctorat de M. IRA Mamadou sont également financés par le PROGEVAL. Nous adressons nos sincères remerciements à ces partenaires techniques et financiers.

\section{RESUME}

L'objectif de la présente étude a été d'évaluer la productivité du cheptel bovin de la Guinée-Bissau à travers ses paramètres démographiques et de déterminer son niveau d'exploitation à travers les indicateurs démographiques globaux. L'étude a été menée au sein de 60 exploitations réparties entre les 4 plus grandes régions détentrices d'animaux d'élevage. La méthode d'enquête utilisée a été celle des $12 \mathrm{MO}$. Les résultats ont révélé une très grande proportion de femelles (70\%) par rapport aux mâles (30\%) et parmi ces femelles, plus de la moitié $(55,23 \%)$ sont des reproductrices. L'âge au premier vêlage s'est fait entre 3 et 4 ans et le taux de parturition global a été de 0,70 année $^{-1} \pm 0,05$. Le taux d'avortement a été faible $\left(0,011\right.$ année $\left.^{-1}\right)$ ainsi que le taux de mortalité global $\left(0,075\right.$ année $\left.^{-1} \pm 0,007\right)$. En ce qui concerne la gestion des troupeaux, le taux d'exploitation brut a été de 0,096 année ${ }^{-1} \pm 0,007$ et celui d'importation de 0,091 année ${ }^{-1} \pm 0,007$; ce qui a donné un taux d'exploitation nette de 0,005 année ${ }^{-1} \pm 0,005$. Les mâles adultes suivis des mâles sub-adultes ont été les plus exploités. Quant aux indicateurs démographiques globaux qui résument la dynamique et la productivité du troupeau sur l'année, le rendement numérique (RN) a été de 0,17 année ${ }^{-1}$ ou $17 \%$ et le croit net (CN) de 0,162 année $^{-1}$ ou $16 \%$. Le cheptel bissau-guinéen est donc caractérisé d'une part par une structure de type naisseur à dominance femelle avec un fort taux de mise-bas et d'autre part par une sous-exploitation du fort potentiel de production.

(C) 2019 International Formulae Group. All rights reserved

Mots clés: Paramètres démographiques, productivité, bovins, 12 MO, Guinée-Bissau. 


\title{
Demographic parameters and productivity of cattle farms in Guinea-Bissau.
}

\begin{abstract}
The objective of this study was to assess the productivity of Guinea-Bissau's cattle population through its demographic parameters and to determine its level of exploitation through global demographic indicators. The study was conducted in 60 farms distributed among the 4 largest livestock-holding regions. The survey method used was that of $12 \mathrm{MO}$. The results revealed a very high proportion of females (70\%) compared to males $(30 \%)$ and among these females, more than half $(55.23 \%)$ are breeders. The age at first calving was 3 years and the overall parturition rate was 0.70 year $^{-1} \pm 0.05$. The abortion rate was low $\left(0.011\right.$ year $\left.^{-1}\right)$ as was the overall mortality rate $\left(0.075\right.$ year $\left.^{-1} \pm 0.007\right)$. With regard to herd management, the overall exploitation rate was 0.096 year $^{-1} \pm 0.007$ and the import rate was 0.091 year $^{-1} \pm 0.007$; which gave a net exploitation rate of 0.005 year $^{-1} \pm 0.005$. Adult males followed by sub-adult males were the most exploited. As for the overall demographic indicators that summarize the dynamics and productivity of the herd over the year, the numerical yield or production rate $(\mathrm{RN})$ was 0.17 / animal / year or $17 \%$ and the net growth rate $(\mathrm{CN})$ was 0.162 / animal / year or $16 \%$. The Bissau-Guinean herd is therefore characterized on the one hand by a female-dominated breedertype structure with a high parturition rate and on the other by an under-exploitation of the high production potential.
\end{abstract}

(C) 2019 International Formulae Group. All rights reserved

Keywords: Demographic parameters, productivity, cattle, $12 \mathrm{MO}$, Guinea-Bissau.

\section{INTRODUCTION}

L'économie de la Guinée-Bissau, à l'instar des autres pays d'Afrique de l'Ouest, est dominée par le secteur primaire qui participe à la formation du PIB à environ $43 \%$ (BAD, 2018). L'agriculture y contribue à hauteur de $49,1 \%$ et occupe environ $80 \%$ de la population active (Gassama et Nshimyumuremy, 2018). Le principal produit d'exportation reste les noix de cajou qui représentent $90 \%$ des recettes d'exportations et $60 \%$ des sources de devises (DGE, 2009). L'élevage est la seconde activité économique agricole après les cultures. Il est pratiqué par $88 \%$ de la population rurale (DGE, 2009). De façon générale, le système d'élevage est essentiellement de type extensif agropastoral avec quelques variabilités selon les régions. Le cheptel, relativement important et très diversifié, est composé de bovins, d'ovins, de caprins, de porcins, de volailles et d'asins. Selon le dernier recensement national du cheptel réalisé en 2009 en Guinée-Bissau, le pays compte 1325412 têtes de bovins, 304745 têtes d'ovins, 649083 têtes de caprins, 4355 têtes d'équins, 34770 têtes d'asins, 343680 têtes de porcins et 1482641 têtes de volailles (DGE, 2009).
Cependant, malgré l'énorme potentialité dont dispose la Guinée-Bissau en matière d'élevage (climat favorable, pâturage abondant pendant une grande partie de l'année, disponibilité en eau, ressources génétiques animales adaptées à l'environnement, cheptel important), le sous-secteur de l'élevage ne contribue que pour $3,5 \%$ à la formation du PIB et 7,8\% au PIB agricole (Correia, 2016). Les raisons évoquées à cette faible contribution de l'élevage à l'économie du pays seraient la méconnaissance des potentialités réelles du cheptel et sa sous-exploitation (Correia, 2016).

L'objectif de la présente étude est d'évaluer la productivité du cheptel bovin bissau-guinéen à travers ses paramètres démographiques et de déterminer son niveau d'exploitation à travers les indicateurs démographiques globaux.

\section{MATERIEL ET METHODES Milieu d'étude}

L'étude a été menée dans les quatre plus grandes régions agro-pastorales du pays à savoir les régions de Gabù, Bafata, Oio et Cacheu (Figure1). Ces quatre régions sont 
réparties entre deux zones agro-écologiques définies par FAO-Aquastat (2005) :

- La zone nord-est comprenant les régions de Gabù, Bafata et Oio. Elle est caractérisée par un climat soudanais avec deux saisons bien distinctes: une saison sèche comprise entre novembre et mai et une saison des pluies de juin à octobre. Les précipitations varient entre 1200 et $1500 \mathrm{~mm}$ et sont étalées sur une moyenne de 107 jours. L'évapotranspiration s'élève à $2507 \mathrm{~mm}$ et la température moyenne annuelle est de $27,4{ }^{\circ} \mathrm{C}$. Les sols y sont, pour la majorité, ferralitiques et ferrugineux tropicaux. Cependant, on rencontre des sols hydromorphes dérivés des alluvions maritimes dans les bas-fonds et les bassins des fleuves et rivières. La végétation est constituée d'une savane arborée et de forêts claires parsemées de graminées qui constituent un excellent pâturage naturel pour les animaux. L'élevage est dominé par les ruminants et est associé à la culture du maïs, du riz de plaines, du sorgho, du mil, du coton, de l'arachide et de l'anacardier. Les bovins sont constitués essentiellement des taurins N'Damas Fula et Boenca avec des tailles de troupeaux allant de 35 à des centaines. L'élevage est pratiqué par les populations peuls et mandingues à dominance religieuse musulmane.

- La zone nord-ouest comprenant les régions de Cacheu, Bissau et Biombo. Elle connaît un climat maritime guinéen, modérément pluvieux et chaud, avec $1500-1877 \mathrm{~mm}$ de pluviométrie moyenne sur une moyenne de 112 jours. La température moyenne annuelle est de $26,6{ }^{\circ} \mathrm{C}$ et l'évapotranspiration de $1837 \mathrm{~mm}$. C'est une zone ayant de bonnes possibilités de production agricole diversifiée. Les sols sont argilo-sableux et hydromorphes. La végétation est constituée d'une savane boisée et de forêts denses. L'élevage est dominé par les espèces à cycle court (porcs et volailles). On retrouve également de petits troupeaux de ruminants. Les bovins de la race manjaque sont ceux qui dominent surtout dans la région de Cacheu. Les ruminants et les volailles sont beaucoup plus utilisés pour les rituels traditionnels que la vente au marché. Ce système est pratiqué par les populations animistes telles que les Pepels, les Balantes, les Manjaques, les Dio- las, les Mancanhes et les Bijagos. L'élevage est associé à la culture de riz de bas-fonds, sorgho, mil, arachide, patate douce, manioc et anacardier.

\section{Méthodes d'échantillonnage et d'enquête}

La méthode de collecte des données s'est appuyée sur celle proposée par Lesnoff et al., (2007) pour les enquêtes transversales rétrospectives $12 \mathrm{MO}$ (12 mois). Les enquêtes de terrain ont été menées du 25 mars au 07 avril 2017. Ces enquêtes, ponctuelles dans le temps, ne durent pas plus deux mois (Lesnoff et al., 2013). Elles peuvent être répétées plusieurs années pour étudier la variabilité interannuelle des paramètres démographiques. $\mathrm{La}$ méthode d'enquête $12 \mathrm{MO}$ est une méthode d'enquête transversale rétrospective pour l'estimation des paramètres démographiques d'un cheptel de ruminant domestique (taux annuels de reproduction, de mortalité et d'exploitation) (Lesnoff, 2011). Elle consiste à recenser tous les animaux présents dans le troupeau lors de l'enquête, puis avec l'aide de l'éleveur d'estimer les effectifs d'événements démographiques (mises-bas, mortalité, exploitation et importation) survenues dans le troupeau lors des douze (12) derniers mois précédant l'enquête. Ainsi la démographie complète du troupeau peut être reconstituée pour cette année passée en utilisant la mémoire de l'éleveur. Les paramètres démographiques ciblés dans la méthode 12 MO sont les variables d'état du cheptel (taille du troupeau, structure par sexe, par âge, par race), les taux démographiques annuels et les indicateurs démographiques globaux du cheptel. L'unité de base de l'enquête $12 \mathrm{MO}$ est l'exploitation (ménage et son troupeau de gestion) qui est composée de l'ensemble des animaux gérés par l'exploitation sous sa responsabilité directe, au moment de l'enquête. Il inclut aussi les animaux qui lui ont été confiés (prêts ou contrats salariés) par des personnes externes à l'exploitation. Il n'inclut pas les animaux dont l'exploitation est propriétaire mais confiés à d'autres exploitations (Lesnoff et al., 2007).

Ainsi, au total 60 exploitations ont été retenues, réparties entre 4 régions administratives (les régions de Gabù et Bafata à l'Est et 
les régions d'Oio et de Cacheu respectivement au Centre et à l'Ouest), à raison de 15 exploitations par région. Ces 15 exploitations par région ont été réparties entre 2 secteurs dans chaque région à raison de 8 ou 7 exploitations par secteur. Ces exploitations, dont les troupeaux devaient avoir des tailles comprises entre 5 et 50 têtes, ont été choisies au niveau des villages ou campements. Au total, l'étude a porté sur1885 animaux.

Deux questionnaires ont été administrés : le questionnaire Q1 qui permet de renseigner sur la structure du troupeau et la reproduction lors des douze derniers mois et le questionnaire Q2 qui renseigne sur les entrées et les sorties des animaux ayant eu lieu dans le troupeau lors des douze derniers mois.

\section{Analyses statistiques}

Les données collectées ont été saisies dans la base de données Access 12mo.accdb de l'outil t12mo développée par Lesnoff et al. (2013) puis exportés vers le logiciel $\mathrm{R}$ version 3.3.2 (R Core Team ${ }^{\circledR}, 2016$ ) pour calculer les différents taux des paramètres démographiques. Ces taux ont été calculés grâce aux fonctions de calculs automatiques contenues dans le package R "t12mo" (Lesnoff et al., 2013). Les paramètres démographiques estimés sont :

- Les «variables d'état», qui décrivent l'état du troupeau à un moment donné :

- La taille du troupeau (nombre d'animaux dans le troupeau);

- La structure sexe-âge du troupeau (\% d'animaux par sexe et classe d'âge dans le troupeau);

- Les «taux démographiques annuels», qui décrivent les événements démographiques survenus dans l'année au sein des troupeaux :

- Les «taux naturels», qui se réfèrent aux performances zootechniques du troupeau (reproduction et mortalité naturelle) ;

- $\quad$ Les «taux de gestion », qui se réfèrent aux décisions prises par l'éleveur (exploitation et importation) ;

- Les «indicateurs démographiques globaux », qui résument la dynamique et la productivité du troupeau sur l'année :
- $\quad$ Le taux de croît annuel (CN);

- Les taux de productivité ou rendement numérique (RN).

La taille du troupeau est présentée en moyenne \pm écart-type et la structure sexe-âge en pourcentage. Les taux démographiques annuels et les indicateurs démographiques globaux sont présentés selon 2 quantités distinctes (Lesnoff, 2011), une probabilité $p$ (comprise entre 0 et 1 ) \pm écart-type et un taux instantané $h$ (exprimé en année ${ }^{-1}$ et pouvant être supérieur à 1$) \pm$ erreur standard. La probabilité d'occurrence $p$ de l'évènement est estimée par le rapport de l'effectif d'évènements survenus dans la période (''année dans notre cas) sur l'effectif d'animaux en début de la période. Le taux instantané $h$ d'un évènement (ex : la mortalité) est estimé par le rapport de l'effectif d'évènements (ex : nombres de morts) survenus durant l'unité de temps (l'année dans notre cas) sur le temps total de présence des animaux dans la période (encore appelé temps à risque).

Les taux calculés sont aussi présentés selon le sexe des animaux et la classe d'âges. Les âges des animaux sont en effet regroupés selon trois classes: la classe des juvéniles (JUV, veaux et velles de 0 à 1 an exact), la classe des sub-adultes (SAD, taurillons et génisses d'âge $>1$ an à 4 ans exact) et celle des adultes (ADU, taureaux, bœufs et vaches d'âge $>4$ ans). Les formules de calcul des paramètres démographiques estimés (Lesnoff, 2011) sont :

$h_{p a r}=\mathrm{m}_{\mathrm{par}} / \mathrm{T}$, où $h_{p a r}$ : taux instantané de mise-bas, $\mathrm{m}_{\mathrm{par}}$ : nombre de mise-bas observées dans l'année et $\mathrm{T}:$ le temps total de présence des femelles reproductrices (adultes) dans l'année.

$h_{\text {dea }}=\mathrm{m}_{\text {dea }} / \mathrm{T}$, où $h_{\text {dea }}$ : taux instantané de mortalité naturelle, $\mathrm{m}_{\text {dea }}$ : nombre de morts naturelles observées dans l'année et $\mathrm{T}:$ le temps total de présence des animaux dans l'année.

$h_{a b o}=\mathrm{m}_{\mathrm{ab}} / \mathrm{T}$, où $h_{a b o}$ : taux instantané d'avortement, $\mathrm{m}_{\mathrm{ab}}$ : nombre d'avortements observés dans l'année et $\mathrm{T}:$ le temps total de présence des femelles reproductrices (adultes) dans l'année. 


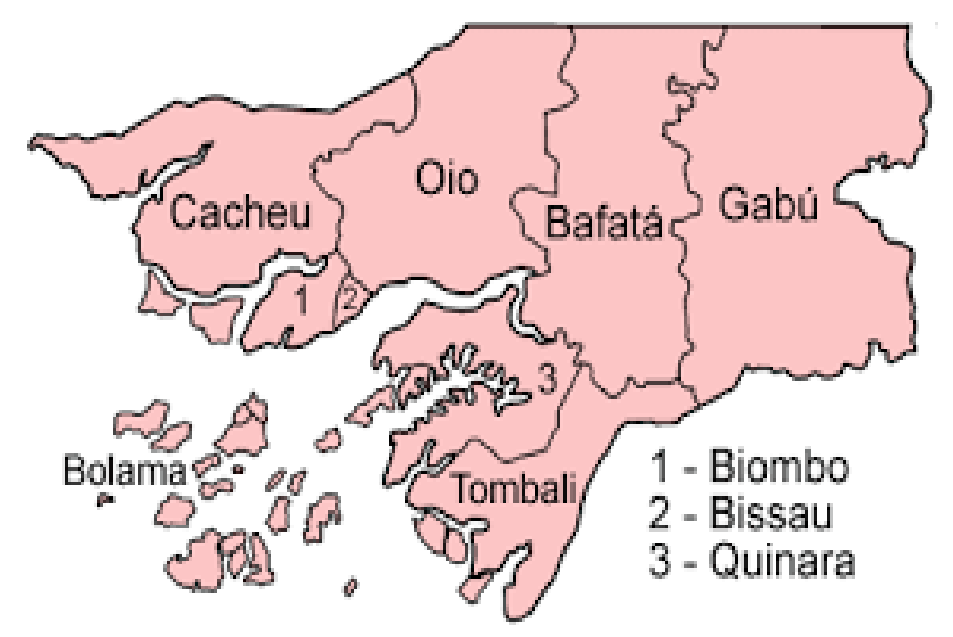

Figure 1: Carte des régions de la Guinée-Bissau.

Le taux de mise-bas moyen sur l'ensemble des carrières des femelles reproductrices a été calculé par la formule de régression linéaire suivante, basée sur l'âge et la parité :

$y=\beta 0+\beta 1 * x$, où $x$ représente l'âge des femelles présentes à la date de l'enquête et $y$ leur parité. La pente de la ligne de régression $\beta 1$ représente le taux de mise-bas moyen annuel et $\beta 0$ est l'intercepte.

Les taux de gestion font référence aux décisions prises par l'éleveur. On distingue le taux d'exploitation (probabilité ou taux instantané qu'un animal soit exploité par abattage, vente, prêt, don, etc.) et le taux d'importation (probabilité ou taux instantané qu'un animal soit importé par achat, prêt, don, etc.).

$h_{\text {off }}=\mathrm{m}_{\text {off }} / \mathrm{T}$, où $h_{\text {off }}$ : taux instantané d'exploitation brute, $\mathrm{m}_{\text {off }}$ : nombre d'animaux exploités observés dans l'année et $\mathrm{T}$ : le temps total de présence des animaux dans l'année.

$h_{\text {int }}=\mathrm{m}_{\text {int }} / \mathrm{T}$, où $h_{\text {int }}$ : taux instantané d'importation, $\mathrm{m}_{\text {int }}$ : nombre d'animaux importés observés dans l'année et $\mathrm{T}:$ le temps total de présence des animaux dans l'année.

L'exploitation nette est la différence entre l'exploitation brute et l'importation.

$h_{\text {offnet }}=h_{\text {off }}-h_{\text {int }}$

Le rendement numérique ( $\mathrm{RN}$ ) qui indique la productivité démographique naturelle du troupeau ou le potentiel effectivement exploitable a été calculé à partir de la variation de stock et du taux d'exploitation selon la formule $\mathrm{RN}=\left(\Delta n+\left(\mathrm{m}_{\text {off }}-\mathrm{m}_{\text {int }}\right)\right) / \mathrm{T}$ où $\Delta n$ représente la variation de stock du troupeau entre le début et la fin de l'année.

Le croit net $(\mathrm{CN})$ qui indique la croissance du troupeau s'obtient par la différence entre le rendement numérique $(\mathrm{RN})$ et l'exploitation nette (EN).

\section{RESULTATS}

\section{Les variables d'état}

Taille moyenne des troupeaux

La taille moyenne des troupeaux enquêtés est de $31 \pm 12$ têtes (valeurs extrêmes : 13 - 50 têtes). La moitié du nombre de troupeaux a un effectif compris entre 21 et 38 têtes. Ces résultats sont illustrés par la Figure 2.

\section{Structure sexe-âge des troupeaux}

Les résultats ont révélé une très grande proportion de femelles (70\%) par rapport aux mâles $(30 \%)$ (Tableau 1) et parmi ces femelles, plus de la moitié $(55,28 \%)$ sont des vaches (Tableau 2).

La répartition des animaux par sexe dans les classes d'âge est présentée dans la Figure 3.

L'ensemble du cheptel est dominé par les femelles adultes $(38,62 \%)$ suivies des génisses $(18,14 \%)$ et des taurillons $(14,22 \%)$. Les taureaux sont très peu au sein des troupeaux $(5,73 \%)$. Quant aux veaux et velles, ils ont représenté respectivement $10,19 \%$ et $13,1 \%$ du cheptel (Tableau 1). 
La répartition des classes d'âge au sein de chaque groupe de sexe est donnée par le Tableau 2 .

Les femelles reproductrices (adultes) représentent plus de la moitié $(55,28 \%)$ des femelles et les génisses en font le quart $(25,97 \%)$. Chez les mâles, les adultes représentent $19,02 \%$ tandis que les taurillons (SAD) font près de la moitié $(47,18 \%)$ de la population mâle et les veaux $33,8 \%$.

En outre, l'âge moyen des femelles était de 4,5 ans $\pm 3,7$ et celui des mâles de 2 ans \pm 2 . Quant à l'âge maximal à la réforme, il a été de 18 ans chez les femelles et de 14 ans chez les mâles.

\section{Taux démographiques annuels \\ Taux naturels \\ Taux de mise-bas et d'avortement}

Des mises-bas (premier vêlage) ont été enregistrées dans la classe d'âge 3 à 4 ans avec un taux de 0,40 année $^{-1} \pm 0,04$ soit $33 \%$. Cependant c'est véritablement à partir de 4 ans que le taux de parturition commence à croitre de façon substantielle ( $h=0,60$ année $^{-1}$ $\pm 0,05$ soit $45 \%$ ) (Figure 4). L'âge maximal d'activité reproductive des femelles s'est fait à partir de 15 ans avec un taux de parturition de 0,58 année $^{-1} \pm 0,19$ soit $44 \%$. Ainsi, la carrière reproductive des femelles s'est étalée sur 12 ans. En outre, sur la période des 12 derniers mois, les vaches ont présenté un très fort taux de mise-bas global estimé à 0,70 année $^{-1}$ avec un âge moyen à la mise-bas compris entre 9 et 10 ans. Ce qui signifie qu'une vache présente toute l'année dans le cheptel a eu en moyenne 0,70 mise-bas. En outre, l'intervalle moyen entre mises-bas (IVV) a été de 521,43 jours soit environ 1 an 5 mois.

La Figure 5 donne la distribution des mises-bas survenues sur toute la carrière des femelles reproductrices par la méthode de régression linéaire.

Ces résultats confirment ceux obtenus durant les 12 derniers mois. En effet, les mises-bas débutent à partir de 3 ans révolus jusqu'à 18 ans révolus et la grande partie de ces mises-bas a eu lieu entre 4 ans révolus et 10 ans révolus. Cependant, le taux de misebas moyen sur l'ensemble des carrières des femelles reproductrices est largement en deçà de celui obtenu sur les 12 derniers mois $(0,42$ année ${ }^{-1}$ contre 0,70 année $^{-1}$ ). La plus forte parité (7) est détenue par une vache de 18 ans révolus.

Par ailleurs, très peu d'avortement se sont produits au sein des troupeaux durant l'année ( $\mathrm{h}=0,011$ année $^{-1}$ soit $\left.1 \%\right)$.

\section{Taux de mortalité}

Le taux de mortalité global a été de 0,075 année $^{-1} \pm 0,007$ soit $7,1 \%$. Le Tableau 3 synthétise la mortalité suivant les différents groupes de classes d'âges. Les plus forts taux de mortalité ont été observés chez les juvéniles (veaux et velles de moins de 1 an).

En outre, selon le sexe, les velles ont enregistré le plus fort taux $\left(0,187\right.$ année $^{-1} \pm$ 0,031 soit $17 \%$ ). Les taux de mortalité des génisses et taurillons étaient pratiquement les mêmes (respectivement 0,11 année $^{-1} \pm 0,017$ soit $10,42 \%$ et 0,10 année $^{-1} \pm 0,019$ soit 9,52\%). Quant aux mâles adultes, ils ont enregistré un taux de 0,021 année $^{-1} \pm 0,015$ soit $2 \%$, qui a été pratiquement le double de celui des vaches $\left(0,012\right.$ année $^{-1} \pm 0,004$ soit $\left.1,2 \%\right)$ (Figure 6).

\section{Taux de gestion}

Les taux d'exploitation brute globale, d'importation globale et d'exploitation nette obtenus sont consignés dans le Tableau 4.

Le taux d'exploitation brute globale a été de 0,096 année $^{-1} \pm 0,007$. Quant au taux d'importation globale, il a été de 0,091 année ${ }^{1} \pm 0,007$. L'exploitation nette qui est la différence entre l'exploitation brute et l'importation a été de $0,005 \pm 0,002$ autant en taux instantané qu'en probabilité.

Le Tableau 5 résume les taux d'exploitation nette suivant le sexe et les classes d'âge.

Suivant le sexe et les classes d'âges et évalué en exploitation nette, les mâles adultes suivis des mâles sub-adultes ont été les plus exploités avec des taux respectifs de $0,139 \pm$ 0,039 et $0,098 \pm 0,019$. Les génisses et les vaches ont été très sous-exploités avec des taux respectifs de $0,008 \pm 0,005$ et $-0,058$ année ${ }^{-1}$.

\section{Les indicateurs démographiques globaux}

Pour l'ensemble des troupeaux enquêtés, le rendement numérique $(\mathrm{RN})$ a été de 0,17 année $^{-1}$ soit $17 \%$ c'est-à-dire 0,17 ani$\mathrm{mal} / \mathrm{an}$ et le croit net $(\mathrm{CN})$ de 0,162 année $^{-1}$ soit $16 \%$. 


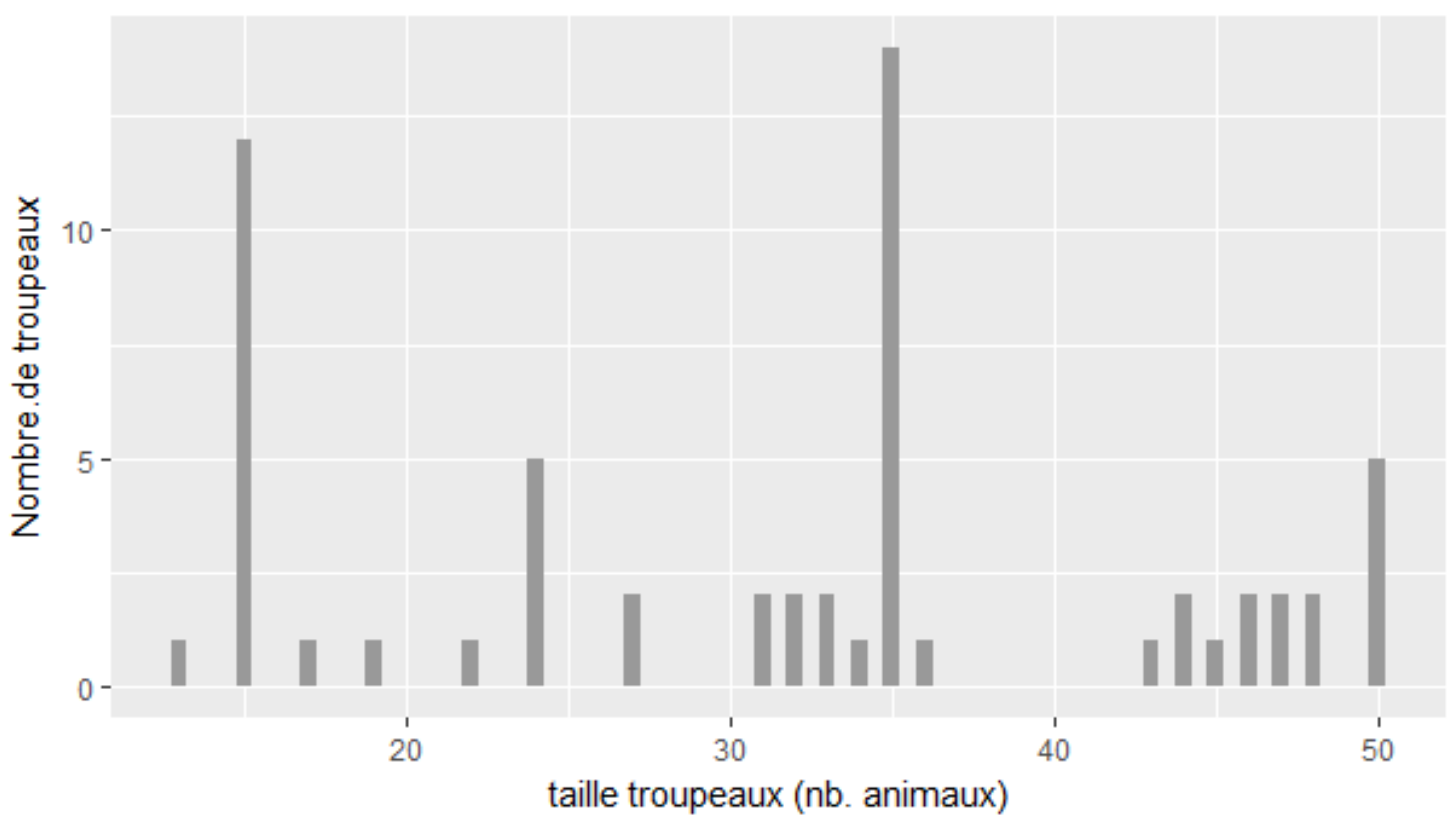

Figure 2: Répartition des troupeaux suivant leurs tailles.

Tableau 1 : Structure sexe-âge du troupeau.

\begin{tabular}{lcccccc} 
& F.JUV & F.SAD & F.ADU & M.JUV & M.SAD & M.ADU \\
\hline $\begin{array}{l}\text { Fréquence sur l'ensemble du } \\
\text { troupeau (\%) }\end{array}$ & 13,1 & 18,14 & 38,62 & 10,19 & 14,22 & 5,73 \\
\hline
\end{tabular}

F.JUV: velles F.SAD: génisses F.ADU: vaches M.JUV:veaux M.SAD: taurillons M.ADU: taureaux.

Tableau 2 : Proportion des classes d'âge intra-sexe.

\begin{tabular}{llcl} 
& \multicolumn{3}{c}{ Classes d'âge } \\
\hline Sexe & JUV & SAD & ADU \\
\hline Femelle (\%) & 18,75 & 25,97 & 55,28 \\
Male (\%) & 33,8 & 47,18 & 19,02 \\
\hline
\end{tabular}

JUV :veaux et velles $\quad S A D$ : taurillons et génisses $\quad A D U$ : taureaux et vaches

Tableau 3 : Mortalités par classe d'âge.

\begin{tabular}{lllll}
\hline Classe d'âge & h & se.h & \% & sd \\
\hline JUV (veaux et velles) & 0,147 & 0,021 & 13,7 & 0,02 \\
SAD (taurillons et génisses) & 0,106 & 0,013 & 10 & 0,01 \\
ADU (taureaux et vaches) & 0,013 & 0,004 & 1,3 & 0 \\
\hline
\end{tabular}

h: taux instantané se.h:erreur standard \%:pourcentage sd:écart-type. 


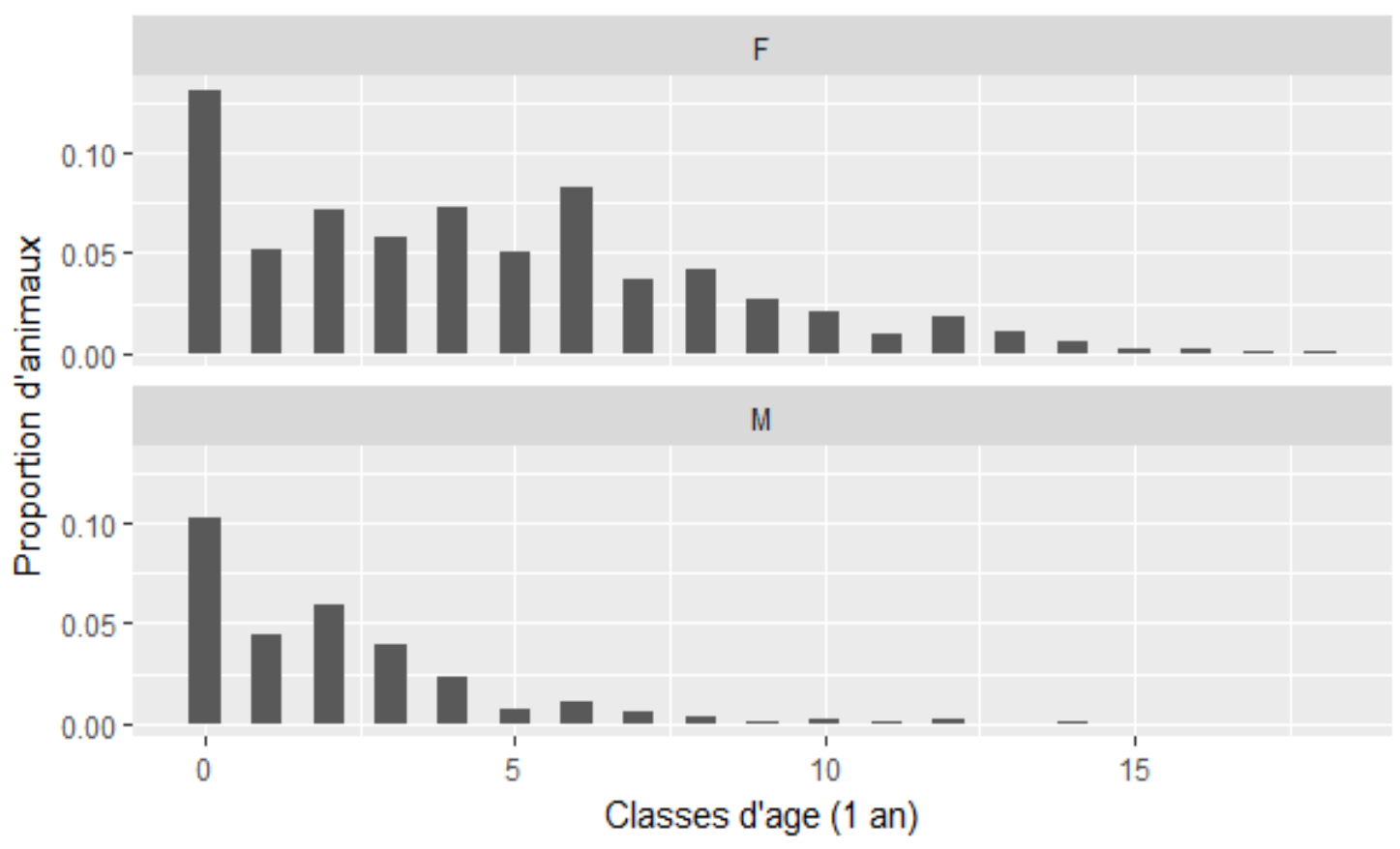

Figure 3: Structure sexe-âge des troupeaux.

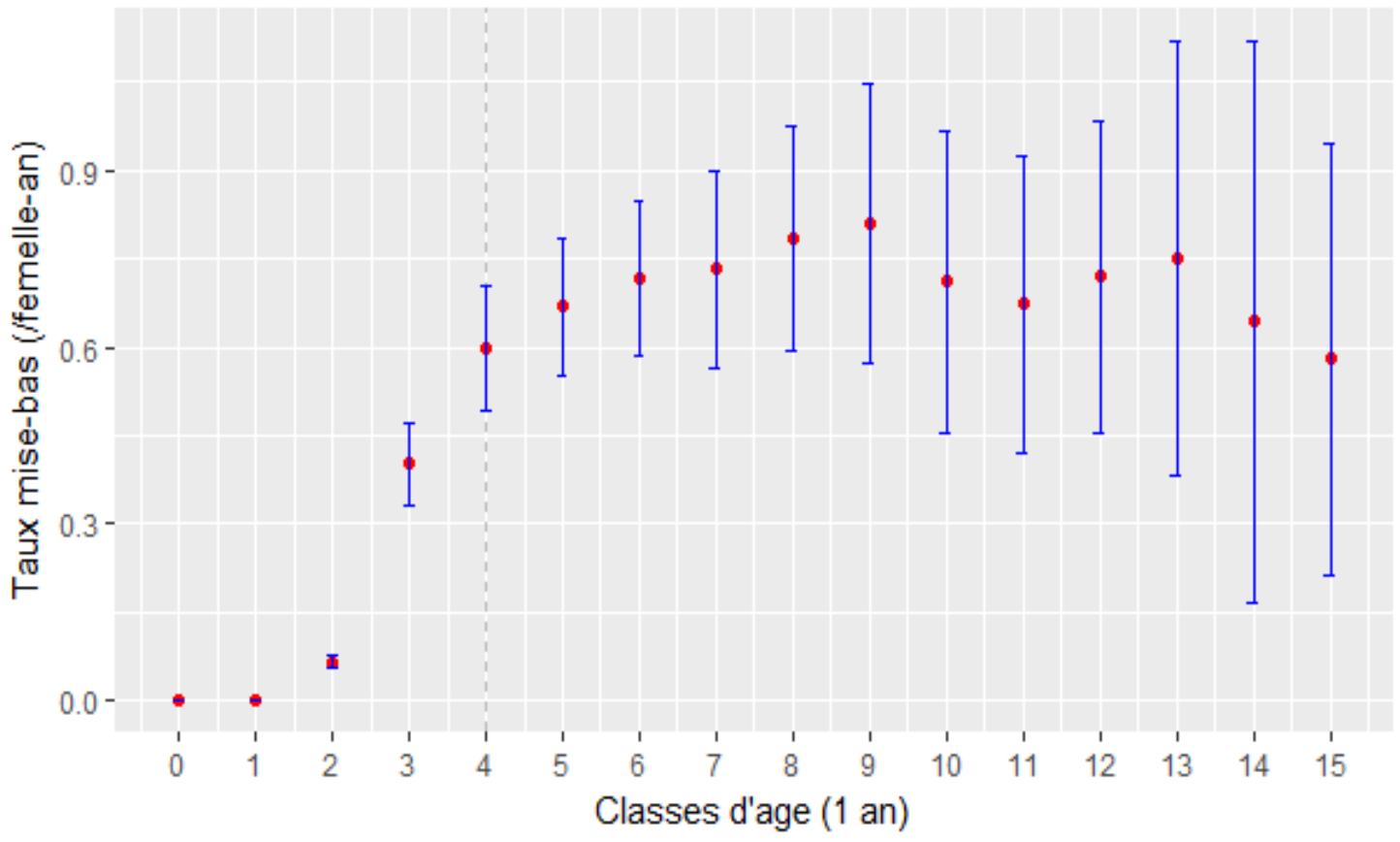

Figure 4: Distribution des mises-bas par classe d'âge sur les 12 derniers mois. 


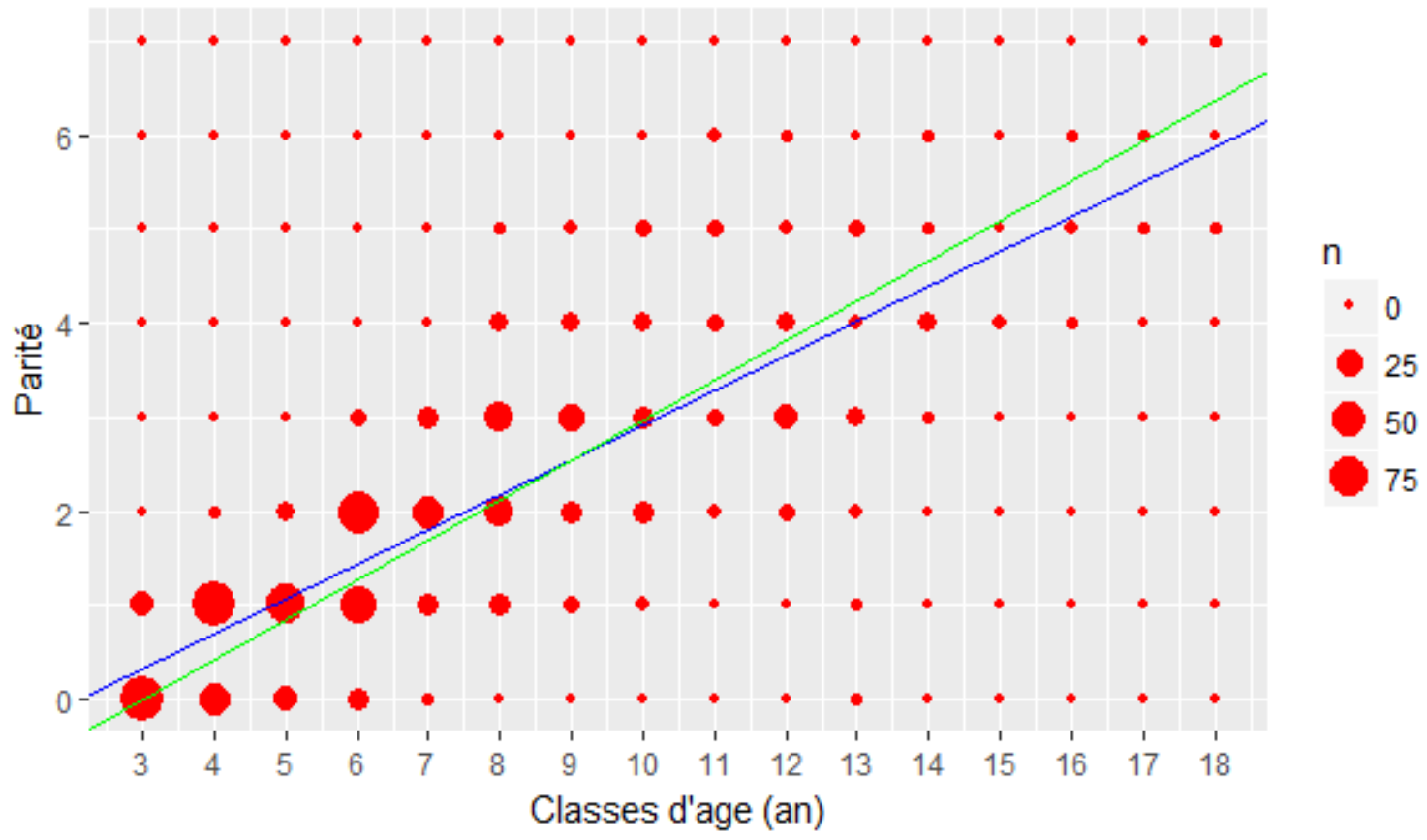

Figure 5 : Distribution des mises-bas par classe d'âge sur toute la carrière des femelles reproductrices.

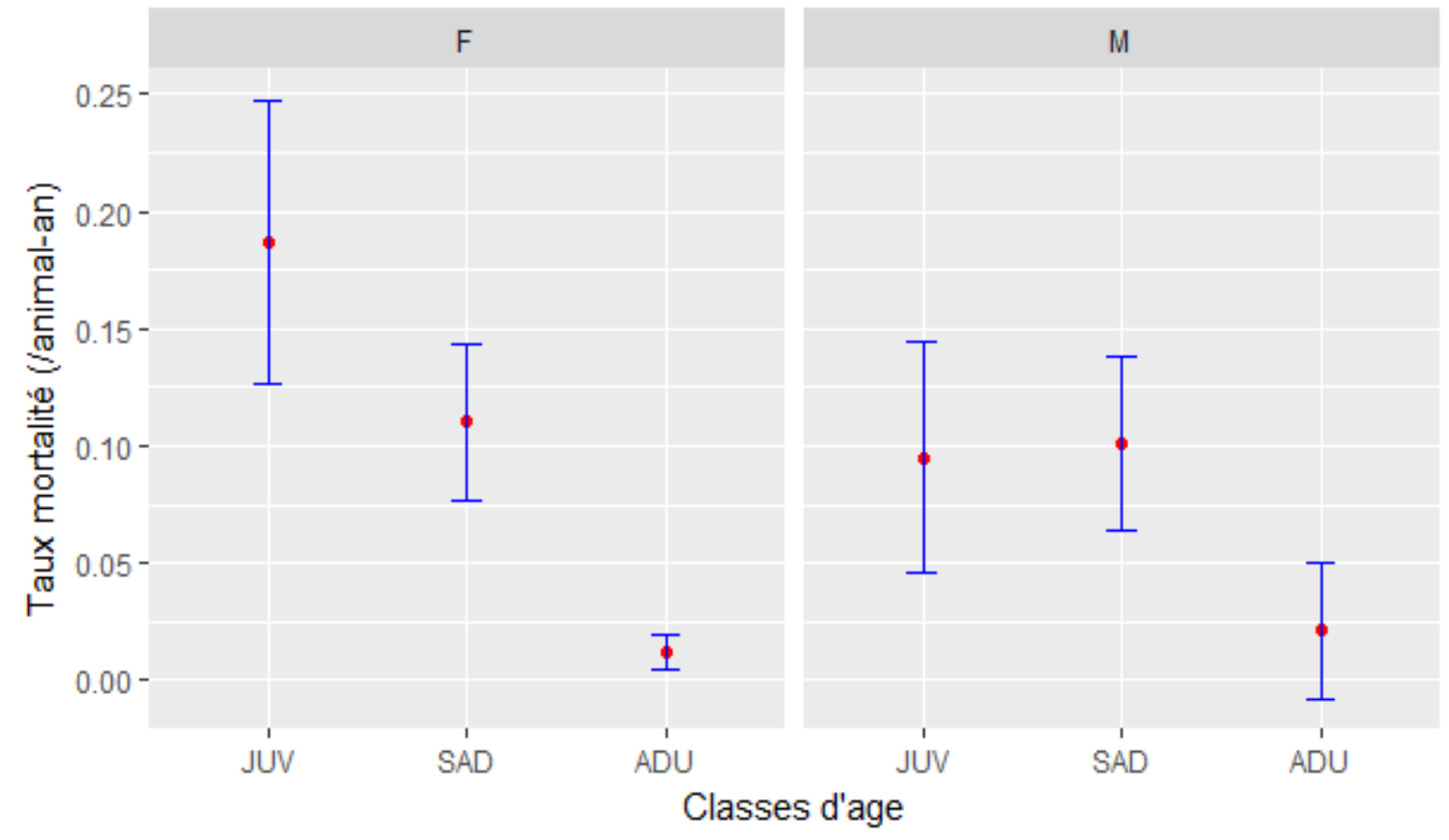

Figure 6: Distribution des mortalités suivant le sexe et la classe d'âge. 
Tableau 4 : Taux d'exploitation brute global, d'importation globale et d'exploitation nette.

\begin{tabular}{lcc}
\cline { 2 - 3 } & $\mathbf{h}\left(\mathbf{a n n e ́}^{\mathbf{- 1}}\right)$ & se.h \\
\hline Exploitation brute globale & 0,096 & 0,007 \\
\hline Importation globale & 0,091 & 0,007 \\
\hline Exploitation nette & 0,005 & 0,002 \\
\hline h: taux instantané se.h: erreur standard & &
\end{tabular}

Tableau 5: Taux d'exploitation nette suivant le sexe et les classes d'âge.

\begin{tabular}{lcccc}
\cline { 2 - 5 } & Taureaux & Taurillons & Génisses & Vaches \\
\hline $\begin{array}{l}\text { Exploitation nette } \\
\left(\text { année }^{-1} \pm \text { se.h) }\right.\end{array}$ & $0,139 \pm 0,039$ & $0,098 \pm 0,019$ & $0,008 \pm 0,005$ & $-0,058$ \\
\hline
\end{tabular}

\section{DISCUSSION}

\section{Taille et structure des troupeaux}

La taille moyenne des troupeaux enquêtés (31 \pm 12 têtes) est comprise dans l'intervalle des tailles moyennes des troupeaux sub-sahariens obtenu par Otte et Chilonda (2002) qui est de 15 à 157 têtes.

La structure des troupeaux, indicateur pertinent pour l'étude de leur dynamique, suit également la tendance observée dans la majorité des troupeaux subsahariens, c'est-à-dire de l'ordre de $1 / 3$ mâles et $2 / 3$ femelles (Hourcade, 2010 au Zimbabwé ; Jorat, 2011 au Sénégal ; Toko et al., 2016 au Bénin; Lesnoff et al., 2006 au Niger ; Somda et al., 2005 en Gambie ; Ba et al., 2011 au Mali ; et Assobga et al., 2017 au Bénin). Ce type de structure est caractéristique des troupeaux naisseurs dont le but est l'augmentation de la taille des troupeaux par les multiples naissances. En outre, la proportion élevée de femelles adultes dans l'ensemble des troupeaux $(38,62 \%)$ et aussi celle des vaches parmi les femelles $(55,28 \%)$ traduisent la stratégie d'élevage mise en place qui est d'assurer la croissance et la pérennité des troupeaux. Cette forte proportion de femelles reproductrices traduit la spécialisation des troupeaux dans la production de veaux et du lait. Ainsi, ces résultats confirment l'accroissement rapide du nombre de bovins du pays qui est passé du simple (615000 têtes) en 2006 au double (1325412 têtes) en 2009 (DGE, 2009). Ceci a été rendu possible grâce à une multiplication du nombre de naissance qui s'est traduit en augmentation du nombre de veaux $(23,29 \%)$, proportion supérieure à celles rapportées par Hourcade (2010) au Zimbabwé, Jorat (2011) au Sénégal et Sounon (2012) qui étaient respectivement de 19,8\%, $18,5 \%$ et $16 \%$. La deuxième forte proportion de classe sexe-âge représentée par les génisses $(18,14 \%)$ traduit une stratégie de remplacement intra-troupeau des vieilles reproductrices réformées et par là le renouvellement des troupeaux. La faible proportion des mâles adultes (5,73\%) s'explique par le fait qu'ils ne sont pas conservés assez longtemps et en grand nombre dans les troupeaux. Seuls quelques taureaux sont gardés pour assurer le cycle de reproduction des troupeaux. En effet, la majorité des mâles sont exploités à partir de 2 ans pour l'abattage ou la vente d'où l'âge moyen des mâles de 2 ans \pm 2 obtenu dans cette étude. Ces résultats sont similaires à ceux rapportés par Toko et al. (2016), Jorat (2011) et Diawara (2017) qui ont obtenu respectivement des proportions de mâles adultes de $6 \%, 4 \%$ et $6 \%$. En outre, les taureaux reproducteurs sont reformés vers 9 ans, c'est-àdire engraissés et vendus aux bouchers. Quant aux femelles, elles sont conservées beaucoup plus longtemps au sein des troupeaux avec un âge moyen compris entre 9 et 10 ans pour les vaches; leur réforme a lieu entre 10 et 18 ans. Les éleveurs conservent de vieilles vaches reproductrices afin de maintenir la croissance de leur troupeau mais aussi, dû au fait que les vieilles vaches sont beaucoup plus adaptées à 
leur environnement d'élevage et résistent mieux aux épizooties. L'élevage traditionnel (transhumant ou sédentaire) est ainsi caractérisé par une conservation de vaches productives dans les troupeaux de reproduction (Youssao et al., 2000). Manoli (2012) a obtenu sensiblement les mêmes résultats dans la commune de Téssékré au Sénégal avec des âges à la réforme des femelles (ARF) et des mâles (ARM) respectivement de 13-14 ans et 9-10 ans, de même que Hourcade (2010) au Zimbabwe dont l'ARF a été de 14-15 ans et l'ARM 9-10 ans. L'ARM obtenu par Jorat (2011) a été inférieur au notre (7ans) tandis que l'ARF est dans le même ordre de grandeur que le nôtre (>10 ans).

\section{Taux démographiques naturels Mises-bas et Avortement}

L'âge moyen au premier vêlage obtenu (3-4 ans avec $\mathrm{h}=0,60$ année $\left.^{-1}\right)$ dénote de la précocité des génisses du cheptel bissauguinéen. Cela s'expliquerait par une pratique d'élevage favorisant une mixité des mâles reproducteurs et des génisses et la disponibilité pendant une longue période de l'année des ressources alimentaires (pâturage et résidus de récolte). Adamou-N'Diaye et al. (2002) rapportent le même âge moyen au premier vêlage de 3 ans avec des bovins Borgou au Bénin, de même que Sokouri et al. (2010) et YapiGnaoré et al. (1996) en Côte d'Ivoire sur des N'Dama. Toutefois, nos résultats diffèrent de ceux rapportés par Jorat (2011) au Sénégal sur des Zébus Gobra, Ezanno et al. (2002) sur des N'Dama et Lawal-Adebowale (2012) sur des zébus Goudali au Nigéria avec des âges de première mise-bas variant de 4 ans à 5 ans. Quant au taux de mise-bas, le fort taux obtenu sur la période des 12 derniers mois traduit la vitalité de ces troupeaux. Ainsi, toutes les vaches présentes toute l'année dans le cheptel ont eu en moyenne 0,70 mise-bas. L'une des explications qu'on peut donner à ce fait est la disponibilité des ressources alimentaires sur une grande partie de l'année due à une bonne pluviométrie. En effet le pays est l'un des plus arrosés de la sous-région avec des précipitations annuelles allant de 1200 à plus de $2500 \mathrm{~mm}$ d'eau (FAO-Aquastat,
2005). Avec des sols assez riches le pâturage et les résidus de récolte y sont abondants et variés. Sous les tropiques, les taux de misebas dépendent fortement de la disponibilité en fourrage et de l'état de santé des troupeaux (Ezanno et al., 2002). Le taux de mise-bas obtenu en Guinée-Bissau est proche de ceux (entre 0,6 et 0,7 année ${ }^{-1}$ ) de Hourcade (2010) au Zimbabwé sur des zébus Mashona et de Toko et al. (2016) et Alkoiret et al. (2010) sur des Borgous au Bénin. Jorat (2011) au Sénégal sur des zébus Gobra, Lawal-Adebowale (2012) au Nigéria sur des zébus Goudali, Ezanno et al. (2002) au Sénégal sur des taurins N'Dama, Ba et al. (2011) au Mali sur des zébus et N'Dama, Ejlertsen et al. (2011) au Sénégal, Sounon (2012) au Bénin, Savadogo (2017) sur des zébus au Burkina avaient tous enregistré des taux de mise-bas inférieurs, compris entre 0,32 et 0,57 année ${ }^{-1}$. Cette différence s'explique par le fait que les zébus et les taurins n'ont pas les mêmes aptitudes de reproduction. Toutefois, des auteurs comme Akouango et al. (2010) sur des N'dama au Congo et Assogba et al. (2017) sur des lagunaires au Bénin ont rapporté des taux supérieurs à ceux de la présente étude, compris entre 0,75 et 0,94 année ${ }^{-1}$.

Par ailleurs, le taux de mise-bas moyen sur l'ensemble des carrières des femelles reproductrices a été largement inférieur à celui obtenu sur les 12 derniers mois $\left(0,42\right.$ année $^{-1}$ contre 0,70 année $\left.e^{-1}\right)$. Ceci s'explique par les variations interannuelles des taux démographiques sous les tropiques dues aux conditions climatiques, alimentaires, et sanitaires. Jorat (2011) a enregistré un taux de 0,58 année $^{-1}$ sur l'ensemble des carrières des femelles reproductrices Gobra dans le Ferlo au Sénégal. Cependant, comparativement à notre échantillon, Jorat (2011) a estimé ce taux sur un échantillon de femelles âgées de 4 à 10 ans, période de reproduction maximale, alors que notre intervalle d'âge est beaucoup plus large, 3 à 18 ans. Notre période de reproduction maximale corrobore celle de Jorat (2011). En effet, au-delà de 10 ans nous enregistrons des taux de mise-bas assez élevés mais avec des écart-types importants. 
Il est important de noter que les avortements ont été rares dans le cheptel lors des 12 derniers mois avec un taux de 0,011 année ${ }^{-1}$ soit $1 \%$. Ce résultat est largement inférieur à ceux obtenus par Assogba et al. (2017), Diawara et al. (2017), Hourcade (2010), Alkoiret et al. (2010) et Sokouri et al. (2010) qui étaient respectivement de 4 à $11 \%, 2,43 \%$, $16 \%, 9,3 \%$ et $3 \%$ dans des populations de lagunaires au Bénin, de zébus Peuhls au Mali, de taurins N'Dama au Bénin, de zébus Mashona au Zimbabwé et de N'Dama en Côte d'Ivoire et de 3,9\% chez les bovins Borgou au Bénin. Les causes de ces avortements restent souvent non déterminées en l'absence d'un diagnostic réalisé par un vétérinaire ou un laboratoire. Cependant, certaines pathologies comme la brucellose sont le plus souvent incriminées.

\section{Mortalité}

Le taux de mortalité global enregistré a été sensiblement égal à ceux obtenus par Otte et Chilonda (2002) en Afrique subsaharienne, Mavedzenge et al. (2006) au Zimbabwé et Savadogo (2017) au Burkina Faso. Ce taux est très faible par rapport à ceux de $19 \%$ et $23 \%$ enregistrés respectivement en Côte d'Ivoire et au Bénin par Knopf et al. (2004) et Youssao et al. (2000) en élevage extensif. Cependant, Assogba et al. (2017) ont enregistré de très faibles taux de mortalité sur des lagunaires dans le sud du Bénin variant de 0,02 à 0,05 année $e^{-1}$. Ce relatif faible taux de mortalité des bovins bissau-guinéens est la conséquence d'une disponibilité alimentaire (fourrage et résidus de récolte) sur une grande partie de l'année et de la bonne adaptation des races bovines locales à leur environnement (trypanotolérance).

Selon les classes d'âges, les veaux et velles ont été le groupe dont le taux de mortalité a été le plus élevé. Les causes à cela sont d'ordre nutritionnel et sanitaire. En effet, le prélèvement d'une partie du lait pour la consommation humaine a d'importantes conséquences négatives sur la survie des veaux, et surtout si la période d'allaitement coïncide avec celle de soudure où le fourrage et les résidus se font rares. Le manque de sousproduits agro-industriels (SPAI) dans le pays tels que les tourteaux, la mélasse, la drêche pour soutenir les vaches et les jeunes en période de disette accentue cet état de fait. Nos résultats sont en conformité avec ceux obtenus par Ezanno (2002), Corniaux et al. (2012), Sounon (2012), Ba et al. (2011), Lesnoff et al. (2006) et Toko et al. (2016). Toutefois, des taux nettement élevés ont été rapportés par Otte et Chilonda (2002) en Afrique de l'Ouest, Lesnoff et al. (2002) en Ethiopie et Savadogo (2017) au Burkina Faso qui ont enregistré des taux de mortalité juvénile élevés de l'ordre de $20 \%$.

Ejlertsen et al. (2011), Hiernaux (2013), Alkoiret et al. (2010), Ganaba et al. (2002) et Diawara et al. (2017) ont rapporté de très faibles taux de mortalité juvénile de l'ordre de 5\% en moyenne.

Par ailleurs, le très faible taux de mortalité des adultes enregistré $(1,3 \%)$ s'explique par le fait que d'une part étant dans une région bien arrosée, même en saison sèche, les animaux arrivent à obtenir un minimum d'éléments nutritifs dont ils ont besoin du peu de fourrage restant, surtout autour des fleuves où poussent encore quelques graminées. D'autre part, les taureaux et les vaches sont plus résistants aux différentes pathologies de la zone (surtout la trypanosomose) et mieux adaptés à leur environnement. Ezanno et al. (2002) au sud du Sénégal et Ba et al. (2011) au Mali ont rapporté des résultats similaires tandis que Lesnoff et al. (2002) en Ethiopie et Savadogo (2017) au Burkina Faso ont enregistré des taux un peu plus élevés de 3 à $5 \%$, voire 12\% par Sounon (2012) au nord Bénin. $\mathrm{La}$ sous-alimentation, l'absence ou l'insuffisance de prophylaxie sanitaire et médicale sont des facteurs qui augmentent la mortalité en élevage traditionnel (Youssao et al., 2000). Le charbon symptomatique et le charbon bactéridien sont les principales pathologies qui causent la mort des bovins adultes en Guinée-Bissau (Bourzat et al., 2016).

\section{Gestion des troupeaux}

Ces taux d'exploitation, d'importation et le rendement numérique traduisent le mode de gestion des troupeaux par les éleveurs. Les types d'exploitation identifiés dans la zone 
d'étude étaient essentiellement les ventes, les trocs, les abattages, les dons et les prêts. Le taux d'exploitation nette obtenu $(0,5 \%)$ est largement faible par rapport à ceux observés dans d'autres pays africains. En effet, Diawara et al. (2017) au Mali, Jorat et al. (2011) au Sénégal, Ba et al. (2011) au Mali, Hourcade (2010) au Zimbabwe, Pradère (2007) au Mali, Otte et Chilonda (2002), Assani et al. (2015) et Alkoiret et al. (2010) au Bénin ont obtenu des taux compris entre $5,2 \%$ et $24 \%$. Plusieurs causes peuvent expliquer ce faible taux d'exploitation $\mathrm{du}$ cheptel bovin bissauguinéen. On note l'absence d'un véritable marché à bétail et des difficultés de transport des animaux des zones d'élevage (essentiellement à l'Est du pays) vers les grandes villes qui disposent d'abattoirs notamment Bissau la capitale. Par ailleurs, la production et la vente des noix de cajou occupent la majorité des paysans car cette activité est jugée beaucoup plus rentable par rapport à la filière bétailviande. D'autre part, la grande disponibilité en poissons comme source de protéine alternative, et de loin préférés par les habitants, concurrence fortement le marché bétail-viande. Il est aussi à noter que le marché d'exportation du bétail à l'étranger et celui de transformation des produits animaux sont quasi inexistants. A tout cela s'ajoutent les raisons d'ordre social et culturel qui favorisent la thésaurisation du cheptel comme moyen d'épargne et de prestige pour les familles. On y pratique un élevage de capitalisation plutôt qu'un élevage de marché. Le peu d'animaux exploités le sont essentiellement par vente occasionnelle (en fonction des besoins financiers) ou par abattage lors des cérémonies et fêtes religieuses. Avec respectivement des taux de $13 \%$ et $9 \%$, les taureaux et les taurillons sont les plus exploités. En effet, les mâles sont exploités à partir de 1 an révolu et ce de façon exponentielle au fur et à mesure que l'âge augmente. Parmi les mâles adultes, seuls les taureaux reproducteurs sont gardés au sein des troupeaux. Comme dans beaucoup d'autres pays ouest-africains, les génisses et les vaches sont conservées pour la plupart car elles constituent le capital naisseur et laitier du troupeau (Youssao et al., 2000). Les femelles vendues sont généralement de vieilles vaches reformées ou stériles.

Par ailleurs, une productivité numérique élevée assure le renouvellement à terme du troupeau et permet sa croissance, mais permet aussi la vente d'un nombre élevée d'animaux et donc une bonne exploitation du troupeau. C'est donc un paramètre particulièrement important pour caractériser un système d'élevage.

Avec un taux de croit de $16 \%$, le rendement numérique des bovins de la Guinée Bissau a été l'un des plus élevés de la sousrégion (17\%) à cause du faible taux d'exploitation. En effet, les paramètres démographiques globaux obtenus ont été supérieurs à ceux enregistrés par Diawara et al. (2017), Jorat (2011), Ba et al. (2011), Pradère (2007), Otte et Chilonda (2002), et Alkoiret et al. (2010) qui ont obtenu des taux de - 8,8 à 13\% pour le $\mathrm{CN}$ et de 8 à $16 \%$ pour le RN. Seuls les rendements numériques obtenus par Hourcade (2010) et Assani et al. (2015) se rapprochent de la valeur observée en Guinée-Bissau. Assobga et al. (2017) quant à eux, ont obtenu au sud Bénin avec des lagunaires un croit net de $25 \%$ et un rendement de $24 \%$. Ainsi, le rendement numérique obtenu dans notre étude étant largement supérieur à l'exploitation (34 fois), on en déduit que le cheptel bovin bissauguinéen est un cheptel de type naisseur caractérisé par une augmentation du nombre de tête au détriment de l'exploitation qui reste très faible; ce qui explique mieux l'augmentation rapide du cheptel bovin bissau-guinéen qui est passé de 615000 têtes en 2006 à 1325412 têtes en 2009 (DGE, 2009). Les 17\% de la taille des troupeaux que représente le potentiel exploitable sont sous-exploités.

\section{Conclusion}

Le cheptel bovin bissau-guinéen a une structure très dynamique, semblable à celle de la majorité des troupeaux bovins subsahariens. Cette structure est caractérisée par une dominance des femelles de l'ordre de $2 / 3$ et surtout des vaches reproductrices. Ces troupeaux sont essentiellement des troupeaux naisseurs dont le but est d'assurer leur accroissement numérique et de les pérenniser durablement. Les taux de mise-bas sont élevés et les taux de mortalité et d'avortement sont faibles, ce qui a permis de doubler l'effectif du cheptel de 2006 à 2009. Cependant, le très faible taux d'exploitation nette obtenu n'est pas une bonne option pour une valorisation durable de ces ressources et l'atteinte des objectifs de la lettre de politique de développement de l'élevage (LPDE) qui sont entre autres « lutter de façon durable contre la pau- 
vreté en favorisant l'augmentation des revenus des éleveurs, lutter contre l'insécurité alimentaire et la malnutrition et contribuer de façon conséquente à la formation du PIB ». Etant donné que l'étude n'a porté que sur une année, certains résultats obtenus devraient être interprétés avec précaution. Il serait important pour le pays de répéter 2 à 3 autres enquêtes 12 MO similaires afin de prendre en compte non seulement la variabilité saisonnière mais aussi la variabilité interannuelle ou de faire un suivi longitudinal sur 2-3 ans.

\section{CONFLIT D'INTERETS}

Les auteurs déclarent qu'il n'existe aucun conflit d'intérêts lié à la présente étude.

\section{CONTRIBUTIONS DES AUTEURS}

MI est l'auteur principal de l'article. Il a organisé et supervisé la collecte des données, procédé à l'analyse et l'interprétation des données et rédiger l'article. GKD a été l'instigateur principal du projet scientifique de ce présent manuscrit. A cet effet, il a participé à l'organisation de la collecte des données et aussi à l'élaboration du protocole d'analyse des données. Il a supervisé ce présent travail de la collecte des données à la rédaction du manuscrit. MS a aussi contribué grandement à la rédaction de ce présent manuscrit en apportant son expérience dans l'interprétation scientifique des résultats et une révision critique du document. AT, CVY-G et GAO ont eux aussi contribué à la rédaction et à une révision critique du manuscrit et l'ont approuvé. $\mathrm{BD}, \mathrm{JG}$ et $\mathrm{BC}$ ont contribué à l'organisation et à la réalisation de la collecte des données.

\section{REMERCIEMENTS}

Nos remerciements vont également à toute l'équipe de la Coordination nationale PROGEVAL, à toutes les Directions techniques et à tous les éleveurs de la Guinée Bissau qui ont bien voulu participer à cette étude pour leur collaboration.

\section{REFERENCES}

Adamou-N'diaye M, Gbangboche AB, Ogodja OJ, Hanzen C. 2002. Fécondité de la vache Borgou au Bénin : effet de l'âge au premier vêlage sur l'intervalle entre vêlages. Revue. Élev. Méd. Vét. Pays Trop., 55 (2) : 159-163.

Akouango F, Ngokaka C, Ewomango P, Kimbembe E. 2010. Caractérisation mor- phométrique et reproductive des taureaux et vaches N'Dama du Congo. Animal Genetic Resources, 46: 41-47. () Food and Agriculture Organization of the United Nations, 2010. DOI: 10.1017/S2078633610000688.

Alkoiret TI, Awohouedji DYG, Yacoubou AM. 2010. Paramètres démographiques des chep-tels de bovins Borgou et N'Dama à la Ferme d'Elevage de l'Okpara au nord-est du Bénin. Int. J. Biol. Chem. Sci., 4(5): 1657-1666.

Assani, SA, Assogba BCG, Toukourou Y, Alkoiret IT. 2015. Productivity of Gudali cattlefarms located in the commons of Malancity and Karimama extreme north of Benin. Livestock Research for Rural Development, 27. http://www.lrrd.org/lrrd27/7/assa27127.h tml.

Assogba BGC, Assani SA, Alkoiret TI, Youssao AKI. 2017. Demographic parameters of La-gune cattle herds in the Oueme valley in southern Benin. International Research Journal of Natural and Applied Sciences, 4(5): 150-169.

Ba A, Lesnoff M, Poccard-Chapuis R, Moulin CH. 2011. Demographic dynamics and off-takeof cattle herds in southern Mali. Trop. Anim. Health Prod., 43: 11011109. DOI: $10.1007 / \mathrm{s} 11250-011-9808-2$

BAD. 2018. Banque Africaine de Développement : informations clés sur la GuinéeBissau.

https://www.afdb.org/fr/countries/westafrica/guinea-bissau/ Consulté le 22 février 2018.

Bourzat D, Cipriano F, Ichou S. 2016. Rapport de mission d'évaluation PVS de suivi 2015 des services vétérinaires de la Guinée-Bissau, mai 2016. Organisation mondiale de la santé animale (OIE), 12, rue de Prony F-75017 Paris, France. 148 pages.

Corniaux C, Lesnoff M, Ickowicz A, Hiernaux P, Diawara MO, Sounon A, Aguilhon M, Da-walak A, Manoli C, Assani B, Jorat T, Chardonnet F. 2012. Dynamique des cheptels de ruminants dans les communes de Tessékré (Sénégal), Hombori (Mali), Dantiandou (Niger) et Djougou (Bénin), ANR-ECLIS, 41 pages.

Correia F. 2016. Revue des filières Bétail/Viande et Lait et des politiques qui les influencent en Guinée-Bissau. Edi- 
teurs : Niang M, Salla A, Bedane B. Rome : FAO et CEDEAO, 52p. Disponible sur www.fao.org/publications.

DGE. 2009. Synthèse des résultats du recensement national de l'élevage en GuinéeBissau. Rapport définitif. Ministère de l'agriculture, des forêts, de la chasse et de l'élevage. 30 pages.

Diawara MO, Hiernaux P, Mougin E, Gangneron F, Soumaguel N. 2017. Viabilité de l'élevage pastoral au Sahel : Etude de quelques paramètres démographiques des élevages de Hombori (Mali). Cah. Agric., 26 (4): $45006 . \quad$ DOI: https://doi.org/10.1051/cagri/2017039 .

Ejlertsen M, Marshall K, Poole J. 2011. Gestion durable du bétail ruminant endémique d'im-portance mondiale en Afrique de l'Ouest : estimation des paramètres démographiques du cheptel au Sénégal. Rapport de recherche. ILRI, Nairobi, Kenya, 39 p.

Ezanno P, Ickowicz A, Faye B. 2002. Demographic parameters of N'Dama cattle raised under extensive range management conditions in Southern Senegal. Revue Elev. Med. Vet. Pays Trop., 55 (3) :211-219.

FAO-Aquastat. 2005. L'irrigation en Afrique en chiffres, enquêtes, Guinée-Bissau. 8 pages.

Ganaba R, Bengaly Z, Ouattara L. 2002. Calf morbidity, mortality and parasite prevalences in the cotton zone of Burkina Faso. Preventive Veterinary Medicine, 55 (3): 209 - 216. DOI: $10.1016 / \mathrm{S} 0167-$ 5877(01)00287-2.

Gassama K, Nshimyumuremy A. 2018. Perspectives Économiques en Afrique 2018, Guinée Bissau. Banque Africaine de Développement. 11 pages. https://www.afdb.org/fileadmin/uploads/ afdb/Documents/Generic-

Docu-

ments/countrynotes/Guin\%C3\%A9e_Bis sau_note_pays.pdf.

Hiernaux P, Mougin E, Diawara M, Soumguel N, Diarra L. 2013. How much does grazing contribute to herbaceous decay during the dry season in Sahel rangelands? Elevage Climat et Société, ECLiS 3.4. Toulouse, France: Géosciences Environnement Toulouse (GET), 22 pages. [Google Scholar].
Hourcade M. 2010. Estimation des paramètres démographiques des systèmes d'élevage bovins et analyse de la filière viande bovine, dans le Southeast Lowveld (Zimbabwe), Rapport de stage de seconde année Master BGAE-SCIENCES pour l'Environnement (Spécialité Ecologie fonctionnelle et développement durable parcours EPSED Elevage des pays du sud : Environnement, Développement), CIRAD, 59 pages.

Jorat T. 2011. Simulations de dynamiques de cheptels bovins après une sècheresse au sahel en fonction des types d'exploitation pastorales : cas du Ferlo au Sénégal, Rapport de deuxième année Master BGAE-Sciences pour l'environnement (spécialité Ecologie fonctionnelle et développement durable parcours EPSED Elevage des pays du sud : Environnement, Développement), CIRAD, 53 pages.

Knopf L, Komoin-Oka C, Betschart B, Gottstein B, Zinsstag J. 2004. Production and Health Parameters of N'Dama Village Cattle in Relation to Parasitism in the Guinea Savannah of Côte d'Ivoire. Revue Élev. Méd. Vét. Pays Trop., 57 (12): 95-100.

Lawal-Adebowale OA. 2012. Dynamics of ruminants livestock management in the context of Nigerian agricultural system. Chapiter 4, Agricultural and Biological Sciences Livestock Production: 61-80. DOI: http://dx.doi.org/10.5772/52923. Consulté le 17/06/2017.

Lesnoff M, Diedhiou ML, Laval G, Bonnet P, Workalemahu A, Kifle D. 2002. Demographic parameters of domestic cattle in a contagious bovine- pleuropneumonia infected area of Ethiopian highlands. Revue Élev. Méd. Vét. Pays Trop., 55, 139147.

Lesnoff M, Saley A, Adamou K, N'Djaffa H. 2006. Enquête démographique 2006 sur le chep-tel domestique au Niger : sites du Fakara, de Gabi et de Zermou. ICRISAT, Niamey, Niger / DGCD, Bruxelles, Belgique / ILRI, Addis Ababa, Ethiopia.

Lesnoff M, Saley M, Adamou K, N'Djaffa H, Ayantunde A, Gerard B. 2007. 12 MO : Uneméthodologie rétrospective pour l'estimation des paramètres démographiques des cheptels de ruminants domestiques tropicaux. CIRAD-ILRI, 58 pages. 
Lesnoff M. 2011. Démographie et zootechnie tropicales : un lien par les modèles matriciels appliqués aux cheptels de ruminants dans les élevages extensifs, Mémoire de synthèse en vue d'une candidature à une habilitation à diriger des recherches, Université de Montpellier II, $221 \mathrm{p}$.

Lesnoff M, Messad S, Juanès X. 2013. 12 MO: A cross-sectional retrospective method for estimating livestock demographic parameters in tropical smallholder farming systems, CIRAD (French Agricultural Research Centre for International Development), 51p. http://livtools.cirad.fr.

Manoli C. 2012. Le troupeau et les moyens de sécurisation des campements pastoraux: une étude de la gestion des troupeaux de la communauté rurale de Tessekré dans le Ferlo sénégalais. Thèse de Doctorat unique. Ecole doctorale SIBAGHE, Montpellier SupAgro, $247 \mathrm{p}$.

Mavedzenge BZ, Mahenehene J, Murimbarimba F, Scoones I, Wolmer W. 2006. Changes in the livestock sector in Zimbabwe following land reform: the case of Masvingo Province. 117 p.

Otte MJ, Chilonda P. 2002. Cattle and small ruminant production systems in subSaharan Africa: a systematic review. Livestock Information Sector Analysis and Policy Branch, FAO Agriculture Department, Rome, Italy, 98 p.

Pradère JP. 2007. Performances et contraintes de l'élevage au Mali. Version provisoire. Projet d'Appui à l'Agriculture Africaine : Amélioration des politiques agricoles dans les pays d'Afrique de l'Ouest et du
Centre, FIDA, France, OCDE et Hub Rural de Dakar, 73 p.

Savadogo KWI. 2017. Etude des paramètres démographiques du cheptel bovin des élevages péri-urbains de la ville de Bobo-Dioulasso. Mémoire de fin de cycle d'ingénieur du développement rural, Option: Elevage. Institut du Développement Rural / Université Nazi Boni, Bobo-Dioulasso, Burkina Faso. 56 p.

Sokouri DP, Yapi-Gnaoré CV, N'Guetta ASP, Loukou NE, Kouao BJ, Touré G, Kouassi A, Sangaré A. 2010. Performances de reproduction des races bovines locales de Cote d'Ivoire. Journal of Applied Biosciences, 36: 2353-2359.

Somda J, Kamuanga M, Tollens E. 2005. Characteristics and economic viability of milk production in the smallholder farming systems in The Gambia. Agric. Syst., 85: $42-58 . \quad$ DOI: 10.1016/j.agsy.2004.07.011.

Sounon A. 2012. Résultats d'étape de travaux de thèse à Djougou (Bénin), ECLIS.

Toko RC, Adégbidi A, Lebailly P. 2016. Démographie et performances zootechniques des élevages bovins traditionnels au Nord Bénin. Revue Élev. Méd. vét. Pays Trop., 69 (1) : 33-39.

Yapi-Gnaoré CV, Oya BA, Ouattara Z. 1996. Revue de la situation des races d'animaux domestiques de Côte d'Ivoire. Animal Genetic Resources Information. 19 : 99-118.

Youssao AKI, Ahissou A, Touré Z, Leroy PL. 2000. Productivité de la race Borgou à la Ferme d'élvage de l'Okpara au Bénin. Revue Élev. Méd. Vét. Pays Trop., 53 (1) :67-74. 